\title{
UN CAS D'HEMATOME EXTRADURAL CERVICAL NON TRAUMATIQUE
}

\section{A CASE OF NON TRAUMATIC CERVICAL EXTRADURAL HEMATOMA}

N'DA Hermann Adonis 1

KAKOU Médard ${ }^{1}$

DROGBA Kporou Landry ${ }^{1}$

BROALET Maman You Epérance ${ }^{1}$

HAIDARA Aderehime ${ }^{1}$

ZUNON-KIPRE Yvan ${ }^{1}$

KOUAKOU Koffi Fulbert ${ }^{1}$

BONI N'guessan Raymond ${ }^{1}$

VARLET Guy ${ }^{1}$

BAZEZE Vincent ${ }^{1}$

1. Service de Neurochirurgie, CHU de Yopougon 21 BP 632 Abidjan 21 Côte d'Ivoire

E-Mail Contact - N'DA Hermann Adonis : drndah (at) yahoo (dot) fr

Mots-clés: Hématome extradural spontané - Rachis cervical.

Keywords: Spontaneous extradural hematoma, cervical spine

\section{RESUME}

L'hématome extradural cervical spontané est une pathologie rare mais une sévère cause de compression médullaire. II requiert un diagnostic et une prise en charge urgents. Nous en rapportons un cas chez une patiente de 20 ans sans antécédent pathologique, révélé par un syndrome de compression médullaire cervical sévère (grade $A$ de Frankel). Une décompression neurochirurgicale est intervenue avec un délai de $48 \mathrm{H}$ avec comme corollaire de lourdes séquelles. Les auteurs insistent sur l'intérêt d'un diagnostic et d'une prise en charge précoces pour en minimiser les séquelles neurologiques.

\section{SUMMARY}

Spontaneous cervical extradural hematoma is a rare pathology but a severe spinal cord compression cause.We report a case revealed by a severe spinal cord compression (Frankel rank A) in a 20 old female patient without past medical history. A neurosurgical decompression has been performed 48 hours later which result of important after-effects. The authors insist for the early diagnostic and treatment to minimize neurological deficits. 


\section{INTRODUCTION}

L'hématome extradural cervical spontanée ou hématome épidural est une collection hémorragique touchant l'espace épidural [3]. II s'agit d'une pathologie rare se présentant typiquement comme une compression médullaire cervicale aiguë. Elle nécessite un diagnostic et une prise en charge urgents, gages d'un pronostic fonctionnel et parfois vital meilleur. La prise en charge orientée par le grading selon la classification de FRANKEL [9] doit dans tous les cas être urgente pour éviter des séquelles lourdes. Nous présentons un rare cas d'hématome extradural cervical spontané et insistons sur l'intérêt d'un diagnostic et d'une prise en charge précoces.

\section{OBSERVATION}

Madame KA 20 ans sans antécédent particulier a été admise dans notre service au motif d'une quadriplégie installée 48 heures plutôt. L'interrogatoire notait un début brutal marqué par des cervicalgies intenses rapidement associées à un déficit moteur hémicorporel droit se bilatéralisant au bout de quelques minutes aboutissant à une quadriplégie complète.

L'interrogatoire ne retrouvait ni contexte traumatique, ni prise médicamenteuse antérieure. A l'examen physique, la patiente était apyrétique et il existait un déficit moteur complet des 4 membres. On notait également une anesthésie à tous les modes, superficielle et profonde en dessous de C4. Les reflexes ostéotendineux étaient abolis aux 4 membres et il existait une retention urinaire aigue. L'examen permettait de conclure à une compression médullaire cervicale sévère. Cette patiente a été classé au grade $\mathrm{A}$ selon la classification de FRANKEL (tableau 1). Le myéloscanner cervical réalisé en urgence a mis en évidence une coulée épidurale postéro-latérale droite, discrètement hyperdense par rapport à la moelle et comprimant le fourreau dural de la 3 e à la 6è vertèbre cervicale (Fig. 1 et 2). Cette image évocait un processus tumoral épidural.

Une décompression chirurgicale a été réalisée le même jour et il s'agissait d'un volumineux hématome extradural qui a été complètement évacué. La recherche par la suite d'une tare hémorragipare s'est avérée infructueuse. Malgré une rééducation post opératoire intense, cette patiente est restée au grade A de FRANKEL gardant de lourdes séquelles avec un recul de 2 ans.

\section{DISCUSSION}

L'hématome extradural cervical spontané est une pathologie rare $[3,8,9]$ mais une sévère cause de compression médullaire [2]. L'incidence de l'hématome épidural spontané en général, est estimé à 0.1 pour 100.000 habitants et moins d'un pour cent (1\%) de l'ensemble des lésions touchant l'espace épidural [1]. II s'agit du premier cas que nous rapportons en 16 années d'activité.

Divers antécédents telles qu'une coagulopathie, une malformation vasculaire, une maladie de Paget ou la prise d'anticoagulant exposent à la survenue de cette pathologie [9]. La particularité de notre cas est la survenue de cette pathologie sur un terrain jeune indemne de tout antécédent pathologique. Diverses hypothèses ont été évoquées concernant la survenue de ces hématomes extraduraux. Ils pourraient pour certains auteurs provenir de la rupture d'un plexus veineux vertébral [8]. Solero et Fornari, cité par Serizawa et al [8] retrouvent dans $10 \%$ des analyses histologiques d'hématomes épiduraux spontanés une malformation artério-veineuse. Pour Gundry et Heithoff cité par Serizawa et al, la rupture d'une veine épidurale rendue fragile par une hernie discale adjacente est une possible cause de survenue d'un hématome [8].

La majorité des hématomes a une localisation postérieure mais l'hématome en rapport avec une hernie discale a une localisation ventrale par rapport au fourreau dural [8]. Dans notre cas, la localisation de l'hématome a permis d'éliminer une probable rupture veineuse en rapport avec une hernie discale. Les patients atteints de cette pathologie présentent typiquement de façon soudaine des cervicalgies intenses classiquement en coup de poignard et développent rapidement une compression médullaire cervicale [3,9]. Ce caractère brutal, quasi pathognomonique, doit faire évoquer le diagnostic en dehors de toute investigation neuroradiologique.

L'IRM demeure actuellement l'examen de choix pour le diagnostic neuroradiologique de l'hématome extradural cervical [5,8]. II apparaît en hyposignal par rapport à la moelle à T1 et en hypersignal à T2. En dehors des aspects classiques, une lésion inflammatoire ou tumorale peut être mimée par un hématome surtout en cas de rehaussement inhabituel [2]. Cette confusion est très fréquente avec comme corollaire un retard diagnostic et thérapeutique. Dans nos pays où l'IRM est bien souvent absente, l'examen clinique prend une place de choix dans les investigations diagnostiques et couplé au myéloscanner cervical, peut conduire 
au diagnostic. L'existance au myeloscanner d'une lésion hyperdense biconvexe au contact de la dure-mère doit faire evoquer le diagnostic.

D'un point de vue thérapeutique, la décompression neurochirurgicale en urgence est recommandée par la plupart des auteurs $[3,6,8,9]$ comme étant le meilleur traitement. Les grades $A$ et B de FRANKEL doivent faire l'objet d'une chirurgie [9] tandis que les grades $C$ et $D$ peuvent faire l'objet d'un traitement conservateur. Le délais de réalisation de cette chirurgie par rapport à la survenue de l'hématome est un facteur pronostique important conditionnant une éventuelle récupération neurologique [8]. Ainsi, lorsque cette décompression intervient au delà de la $8 \mathrm{e}$ Heure, les chances de régression des lésions sont pauvres [7]. La sévérité des séquelles neurologiques constatées dans notre cas a été le corollaire d'un diagnostic et d'une décompression neurochirurgicale tardive $(48 \mathrm{H})$. Malgré cette évolution, une chirurgie à visée décompressive même tardive doit être réalisée car elle peut être utile [4].

\section{CONCLUSION}

L'hématome extradural cervical est une pathologie rare mais une sévère cause de compression médullaire. Cette pathologie pose des problèmes de diagnostic differentiel avec les myéloradiculites d'installation rapidement progressif ce qui peut en retarder le diagnostic.

Elle impose un diagnostic et surtout une décompression neurochirurgicale urgents chez des patients qui bien souvent ont un contact initial avec les services des urgences médicales.

Cette décompression devra intervenir dans les 8 premières heures afin d'améliorer le pronostic déjà sévère de ces patients. Ce délai très court dans nos conditions de travail doit être le but à atteindre.

Tableau 1 : Classification de FRANKEL

\begin{tabular}{|l|l|}
\hline GRADE A & Déficit sensitivo-moteur complet \\
\hline GRADE B & Déficit moteur complet avec sensibilité résiduelle \\
\hline GRADE C & Déficit sensitivo-moteur résiduel non fonctionnel \\
\hline GRADE D & Déficit sensitivo-moteur résiduel fonctionnel \\
\hline GRADE E & Absence de déficit \\
\hline
\end{tabular}




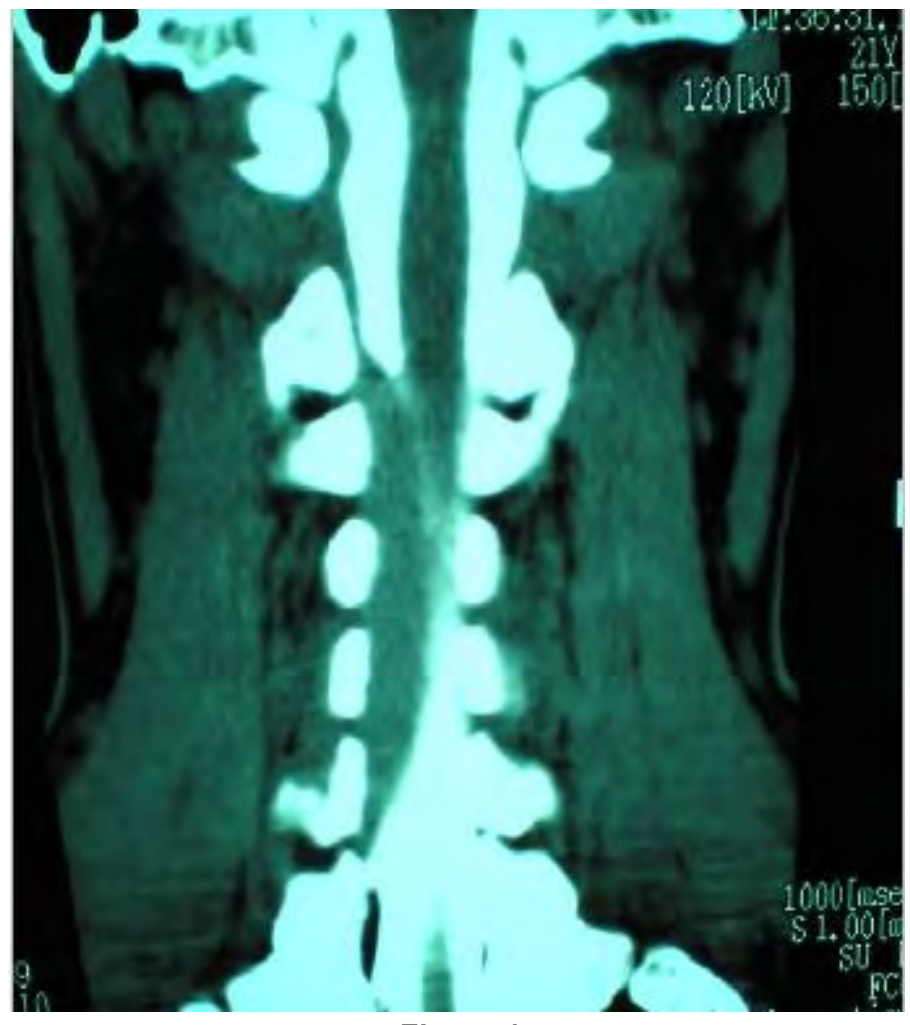

Figure 1

Myéloscanner cervicale en coupe coronale montrant l'hématome

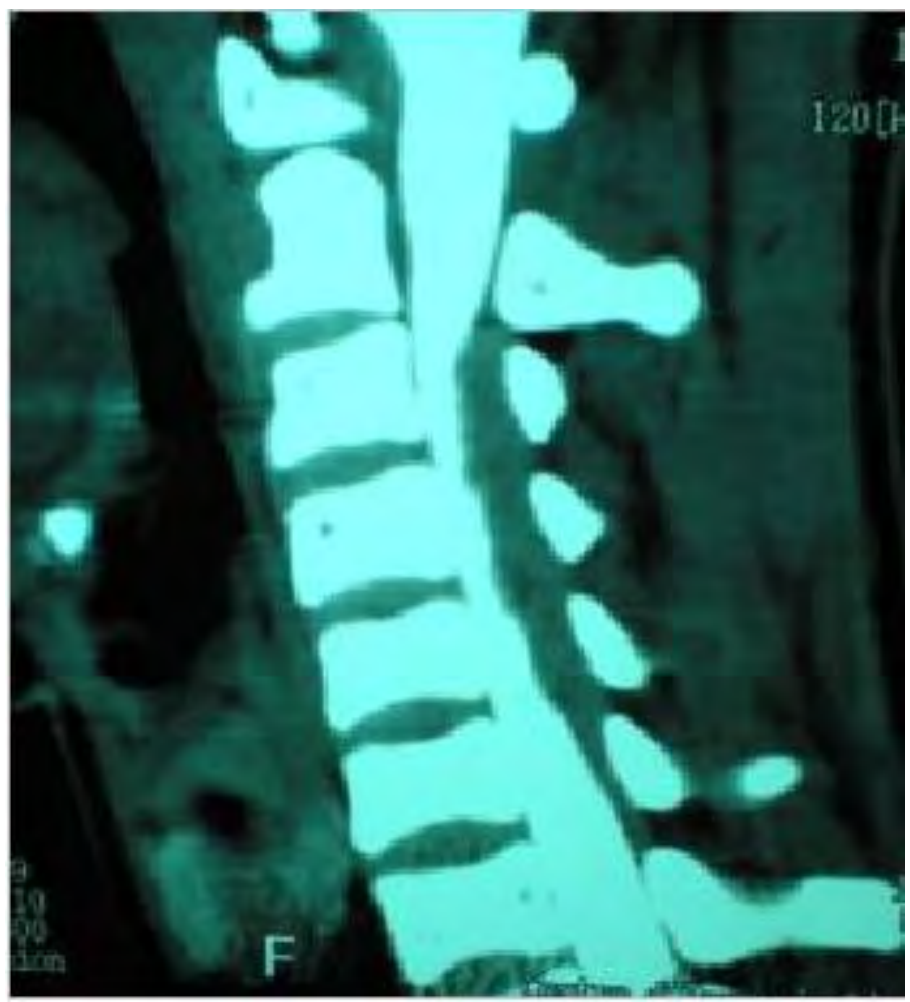

Figure 2

Myéloscanner en coupe sagittale montrant l'hématome 


\section{REFERENCES}

1. BAEK BS, HUR JW, KWON KY, LEE HK. Spontaneous spinal epidura hematoma. J Korean Neurosurg Soc 2008; 44:40-42.

1. CHANG FC, LIRNG JF, CHEN SS, LUO CB. Contrast enhancement patterns of acute spinal epidural hematomas: A report of two cases. AJNR 2003; 24: 366-369.

2. FUIKI MB, SWARNKAR AS, WILLIAM S RL. Acute spontaneous spinal epidural hematoma. AJNR 1999; 20: 1365-1372.

3. GIRARD PL, COURNIL C, DUMAS M. Hématome épidural rachidien spontané. Bull Soc Med. Afr Noire Lang. Fr 1975; 20(3):256-65.

4. KREPPEL D, ANTONIADIS G, SEELING W. Spinal hematoma: A litterature survey with metaanalysis of 613 patients. Neurosurg Rev 2003; 26:1-49.

5. RAVI D, DWARAKANATH S, SATISH R, GOPAL S, VENKATARAMANA NK. Spontaneous Spinal Extradural hematomas. J Clin Neurosci 2006;13:269-272.

6. SCHWARZ SKW, WONG CL, MCDONALD WN. Spontaneous recovery from a spinal epidural hematoma with atypical presentation in a nonagerian. Can J Anesth 2004; 51:557-56.

7. SERIZAWA Y, OHSHIRO K, TANAKA K. Spontaneous resolution of an acute spinal epidural hematoma without neurological deficits. Internal Medecine 1995; 34: 992-994.

8. TORRES A, ACEBES JJ, GABARROS A, LOPEZ L. Spinal epidural hematomas. Prognostic factors in a series of 22 cases and a proposal for management. Neurocirugia 2004; 15:353-359. 